\title{
Intrusion Detection System based on Fuzzy C Means Clustering and Probabilistic Neural Network
}

\author{
Rachnakulhare \\ M.Tech VI sem, \\ BUIT,BU Bhopal
}

\author{
Divakar Singh \\ Head of CSE deptt. \\ BUIT, BU Bhopal
}

\begin{abstract}
Security is always an important issue especially in the case of computer network which is used to transfer personal/confidential information's, ecommerce and media sharing. Since the network is closely related to operating its conditions hence a careful observation \& analysis of network characteristics could describe the state of the network such as network is under specific attack or operating normally. This paper presents an intrusion detection system based on fuzzy $\mathrm{C}$-means clustering and probabilistic neural network which not only reduces the training time but also increases the detection accuracy. The proposed system is tested using KDD99 dataset and the simulation results shows that by selecting effective characteristics and proper training the detection accuracy rate up to $99 \%$ is achievable.
\end{abstract}

\section{Keywords}

Network Security, Neural Network, Intrusion Detection System, Fuzzy C-Means Clustering, KDD99 dataset.

\section{INTRODUCTION}

The Computer Network is designed for easier establishment on network with many facilities but making design like this also exposed it to network attackers and makes it a soft target for intruder hence extra care is required to be taken. To overcome these problems many techniques are proposed one most common is the modification in the protocol (since initially it is designed by considering the performance). Generally the modification in the protocol is toworks for specific attacks only and it may affect the performance also. Another problem with a Protocol modification is all nodes operating in that network must have same protocol or modifications must be compatible with standard one. Another approach which can work independently on any specific node or even on a separate observer unit is generally known as Intrusion Detection System (IDS). An IDS is a device or software application that monitors network or system activities for malicious activities or policy violations and produces reports to a Management Station. Although the IDS do not counter the attack but it can generate alarm. The analysis shows that both systems have their own limitations but a better system can be designed by combining the both algorithms the IDS system can used to initiates the specific modification in protocol and this could be the complete solution for the Intrusion Detection and Prevention System (IDPS).

\section{RELETED WORK}

Because of the importance of the subject already some work has been done some of them which are found most related and useful for making this paper is presented here. Bing Wu et.al. [1] presented great literature on the MANET attacks. Their work gives the detailed explanation of different attacks their behavior and their effect on network characteristics they also explained the security mechanism for some attacks although no simulation and mathematical details are provided. Another text on same topic is presented by Abhay Kumar Rai et.al. [2] the simulation and modeling of the different attacks on MANET using network simulator is explained in KarimKonate et.al. [3] the paper also discussed the protocols and their immunities to different attacks with analytical modeling and mathematical formulation. A graph based approach is proposed by Zhou Mingqiang et al [11] they proposed graph-based intrusion detection algorithm by using outlier detection method that based on local deviation coefficient (LDCGB). Compared to other intrusion detection algorithm of clustering, this algorithm is unnecessary to initial cluster number. Meanwhile, it is robust in the outlier's affection and able to detect any shape of cluster rather that the circle one only. Moreover, it still has stable rate of detection on unknown or muted attacks. Farah Jemili et.al. [4] presented the intrusion detection system based on Bayesian Network (BN). The BN is used to build automatic intrusion detection system based on signature recognition. The goal is to recognize signatures of known attacks, match the observed behavior with those known signatures, and signal intrusion when there is a match. Improved Support Vector Machine (SVM) based IDS model is presented in Jingbo Yuan et.al. [5] the paper discussed the method for improvement of SVM to achieve the higher accuracy. A data preprocessing and removal of similar data to reduce the training data size using $\mathrm{k}$ means clustering presented in [6][12] which shows significant improvement in training time with maintaining accuracy. One important requirement of classification is parameter selection because some of the features may be redundant or with a little contribution to the detection process. Gholam Reza Zargar and PeymanKabiri [7] investigate selection of effective network parameters for detecting network intrusions. The study shows that the major difficulty in develop the system like presented in [5][6][7] is that intrusions signatures changes broadly hence a large training dataset, parameter selection, data filtering and optimal classification is required. Besides mentioned limitation it has a great advantage of better classification without affecting the network performance.

\section{KDD99 DATASET}

This is the data set used for The Third International Knowledge Discovery and Data Mining Tools Competition, which was held in conjunction with KDD-99 the Fifth International Conference on Knowledge Discovery and Data Mining. The competition task was to build a network intrusion detector, a predictive model capable of distinguishing between "bad" connections, called intrusions or attacks, and "good" normal connections. This database contains a standard set of data to be audited, which includes a wide variety of intrusions simulated in a military network environment [9].The KDD99 dataset contains of seven weeks data and the following classes are used for the labeling of each data vectors.

- Denial of Service (dos): Attacker tries to prevent legitimate users from using a service.

- Remote to Local (r21): Attacker does not have an account on the victim machine, hence tries to gain access. 
- User to Root (u2r): Attacker has local access to the victim machine and tries to gain super user privileges.

- Probe: Attacker tries to gain information about the target host.

Subcategories and their Main categories are shown in table 1.

Table 1.List of Intrusions listed in KDD99 dataset.

\begin{tabular}{|c|c|}
\hline Sub-Category & Main-Category \\
\hline back & dos \\
\hline buffer_overflow & u2r \\
\hline ftp_write & r2l \\
\hline guess_passwd & r2l \\
\hline imap & r2l \\
\hline ipsweep & probe \\
\hline land & dos \\
\hline loadmodule & u2r \\
\hline multihop & r2l \\
\hline neptune & dos \\
\hline nmap & probe \\
\hline perl & u2r \\
\hline phf & r2l \\
\hline pod & dos \\
\hline portsweep & probe \\
\hline rootkit & u2r \\
\hline satan & probe \\
\hline smurf & dos \\
\hline spy & r2l \\
\hline teardrop & dos \\
\hline warezclient & r2l \\
\hline warezmaster & r2l \\
\hline & \\
\hline
\end{tabular}

The each data vector of the dataset contains 41 features which can be categorized into four categories:

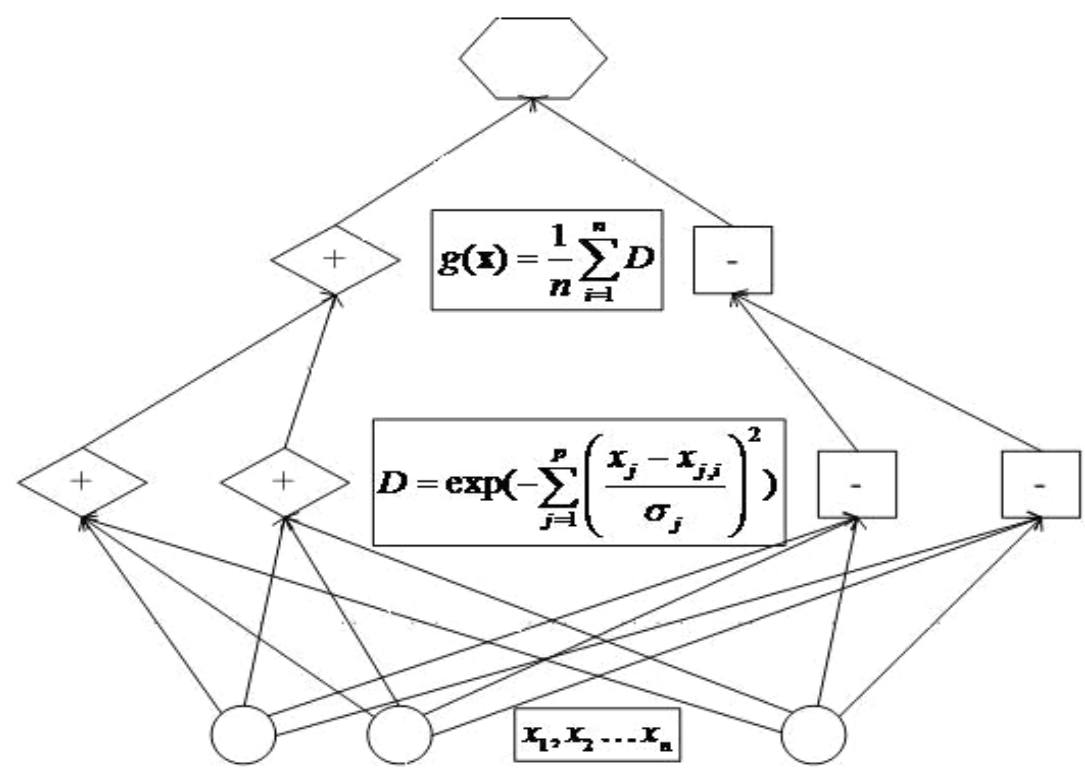
The fourth layer performs a vote, selecting the largest value. The associated class label is then determined [8].

\section{Output layer}

Summation layer

Pattern layer

Fig 1: Structure of PNN [9] 


\section{PROPOSED ALGORITHM}

Inthe proposed system we firstly KDD99 dataset is used to generate the training vectors. Now after collecting all these parameters fuzzy C-means clustering is applied and the data with closer distances are eliminated then this data is used to train the neural network which is later used for detection of Intrusion. The algorithm can be described in detail by following steps:

Step 1: Read the given numbers of samples from KDD99 dataset.

Step 2: Filter selected features from the dataset for further processing.

Step 3: Partition the data into training and testing sets.

Step 4: Cluster the training dataset using the Fuzzy C-means Clustering.

Step 5: Select the fraction of data points from each edge of each cluster.

Step 6: Now train the Probabilistic Neural Network (PNN) using these vectors (data points) with their classification group.

Step 7: Test the trained PNN by the testing dataset.

Step 8: Calculate the performance of the trained system.

\section{BLOCK DIAGRAM OFTHE PROPOSED SYSTEM}

In figure 2 combined frame work the detection system is developed with three modules, namely, pre-processing phase, learning phase and testing phase. Above block diagram of the proposed system can be defined as follows.

- KDD99 dataset: Read the given numbers of samples from KDD99 dataset.

- Pre-processing: In pre-processing module, is to convert the data which is suitable for unsupervised learning by removing the labels from the dataset. Filter selected features from the dataset for further processing.

- Data partitioning: preprocessing data are used to partition into training \& testing sets.

- Fuzzy c- means clustering: Fuzzy clustering plays an important role in solving problems in the areas of pattern recognition and fuzzy model identification. A variety of fuzzy clustering methods have been proposed and most of them are based upon distance criteria. One widely used algorithm is the fuzzy c-means (FCM) algorithm. Cluster the training dataset using the Fuzzy C-means Clustering. Select the fraction of data points from each edge of each cluster.

- Probabilistic neural network: Now train the Probabilistic Neural Network (PNN) using these vectors (data points) with their classification group. PNNs are faster to train and approach the Bayes optimal as the training set increases.

- Testing data: Test the trained PNN by the testing dataset.

- Performance calculation: Calculate the performance of the trained system

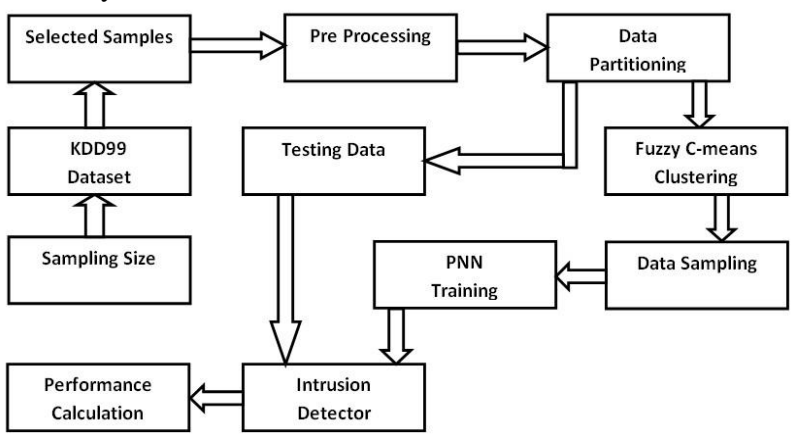

Figure 2: Block diagram of the proposed system.

\section{SIMULATION RESULTS}

The Simulation of the proposed work is performed using MATLAB 7.5 Neural network toolbox in IBM P4 dual core $2.4 \mathrm{Ghz}$ processor with $2 \mathrm{~GB}$ of RAM and windows XP operating system. The results from this simulation are shows in table 2, 2.1 and 3, 3.1.

Table 2.Performance of PNN based System.

\begin{tabular}{|c|r|r|r|r|}
\hline Dataset & \multicolumn{1}{|c|}{ TPR } & \multicolumn{1}{c|}{ TNR } & \multicolumn{1}{|l|}{ FPR } & FNR \\
\hline 1000 & 0.9021 & 0.7934 & 0.2066 & 0.0979 \\
\hline 2000 & 0.7832 & 0.9061 & 0.0939 & 0.2168 \\
\hline 3000 & 0.6837 & 0.9885 & 0.0115 & 0.3163 \\
\hline 4000 & 0.7846 & 0.9035 & 0.0965 & 0.2154 \\
\hline 5000 & 0.9021 & 0.7934 & 0.2066 & 0.0979 \\
\hline
\end{tabular}

Table 2.1Performance of PNN based System.

\begin{tabular}{|c|r|r|r|r|}
\hline Dataset & \multicolumn{1}{|c|}{ Acc. } & \multicolumn{1}{c|}{ Prec. } & Recall & F-meas \\
\hline 1000 & 0.8050 & 0.3417 & 0.9021 & 0.4956 \\
\hline 2000 & 0.8102 & 0.9674 & 0.7832 & 0.8656 \\
\hline 3000 & 0.9540 & 0.8836 & 0.6837 & 0.7709 \\
\hline 4000 & 0.8259 & 0.8914 & 0.7846 & 0.8156 \\
\hline 5000 & 0.9167 & 0.7167 & 0.0833 & 0.0833 \\
\hline
\end{tabular}

Table 3. Performance of Proposed System (Fuzzy C-means and PNN)

\begin{tabular}{|c|r|r|r|r|}
\hline Dataset & \multicolumn{1}{|c|}{ TPR } & \multicolumn{1}{c|}{ TNR } & \multicolumn{1}{l|}{ FPR } & \multicolumn{1}{|l|}{ FNR } \\
\hline 1000 & 0.9680 & 0.9926 & 0.0074 & 0.032 \\
\hline 2000 & 0.9931 & 0.9754 & 0.0246 & 0.0069 \\
\hline 3000 & 0.9594 & 0.9984 & 0.0016 & 0.0406 \\
\hline 4000 & 0.9866 & 0.9798 & 0.0202 & 0.0134 \\
\hline 5000 & 0.9735 & 0.9888 & 0.0112 & 0.0265 \\
\hline
\end{tabular}

Table 3.1 Performance of Proposed System (Fuzzy Cmeans and PNN)

\begin{tabular}{|c|r|r|r|r|}
\hline Dataset & \multicolumn{1}{|c|}{ Acc. } & \multicolumn{1}{c|}{ Prec. } & \multicolumn{1}{|l|}{ Recall } & \multicolumn{1}{|c|}{-meas } \\
\hline 1000 & 0.9900 & 0.9397 & 0.9680 & 0.9536 \\
\hline 2000 & 0.9892 & 0.9931 & 0.9931 & 0.9931 \\
\hline 3000 & 0.9940 & 0.9873 & 0.9594 & 0.9731 \\
\hline 4000 & 0.9898 & 0.9867 & 0.9866 & 0.9866 \\
\hline 5000 & 0.9911 & 0.9734 & 0.9735 & 0.9733 \\
\hline
\end{tabular}




\section{Simulated results of Detection Accuracy:}

Figure 3. Show the graph is plotted between total no. of samplesV/S accuracy. Which show the comparisons between PNN only \& PNN with clustering Detection accuracy? Blue line show PNN only \& Red lines show PNN with fuzzy cmeans clustering. By these comparisons Detection Accuracy performance of PNN with clustering much better than PNN only.

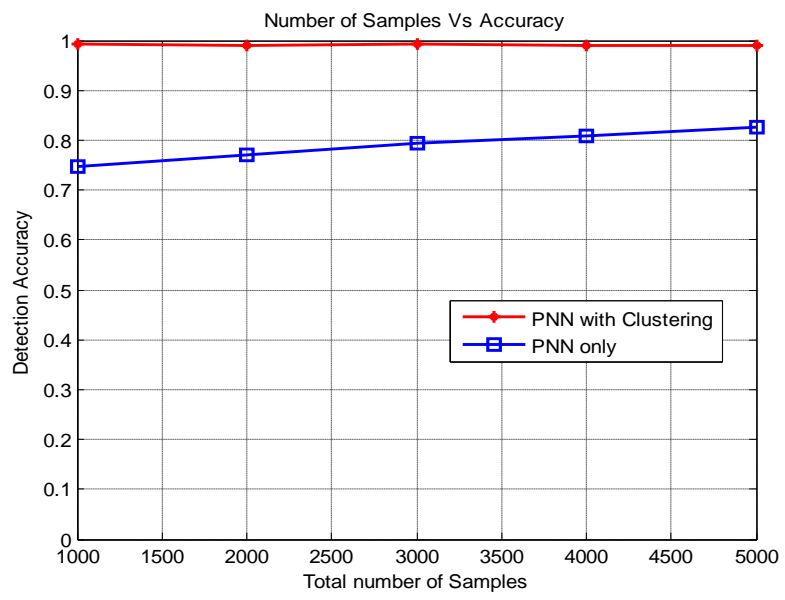

Figure3: Result analysis of PNN with clustering

\section{\& PNN only (Detection Accuracy)}

\section{CONCLUSION}

A simulated resultthe model of the Intrusion detector is presented in this paper is not only capable of attack situation but can also classifying the individual attacks. The Detection accuracy of the system is up to $99 \%$ which is excellent also the algorithm have very low FPR ( $\max 8.3 \%$ ) hence reduces the chances of false alarming. The results also shows that it takes only 0.0075 seconds to identify the intrusion hence fast enough to prevent any loss due to delayed action. Further it could achieve much better performance by increasing the number of samples taken and increasing the number of characteristics parameter selected.

\section{REFERENCES}

[1] Bing Wu, Jianmin Chen, Jie Wu and MihaelaCardei,"A Survey of Attacks and Countermeasures in Mobile Ad Hoc Networks", wireless/mobile network security,2006 Springer.

\section{AUTHOR' BIOGRAPHY}

1. Ms. Rachnakulhare,presently she is a M.tech student of Computer Science \& Engineering, BUIT, Barkatullah University, Bhopal. She Received degree B.E. from UIT RGPV, Bhopal, \& 5 years teaching experience in UG respectively.

2. Dr. Divakar Singh, presently he is Head in Computer Science \& Engineering, BUIT, Barkatullah University, Bhopal. $\mathrm{He}$ received degree B.E., form Barakatullah University Bhopal, M.TECH from RGTU, Bhopal \& Ph.D. in Computer Science \& Engineering. He has research interests in image analysis, image mining, soft computing \& machine learning. He has 12 years of teaching experience in UG and 8 years of PG respectively, also guided more then 35 Mtech Dissertation
[2] Abhay Kumar Rai, Rajiv RanjanTewari and Saurabh Kant Upadhyay,"Different Types of Attacks on Integrated,MANET,InternetCommunication",Internatioal Journal of Computer Science and Security (IJCSS),Aug. 2010.

[3] KarimKonate and Gaye Abdourahime "Attacks Analysis in mobile ad hoc networks: Modeling and Simulation", Second International Conference on Intelligent Systems, Modelling and Simulation, 2011 IEEE.

[4] Farah Jemili, MontaceurZaghdoud and Mohamed Ben Ahmed,"A Framework for an Adaptive Intrusion Detection System using Bayesian Network", 2007 IEEE.

[5] Jingbo Yuan, Haixiao Li, Shunli Ding and Limin Cao "Intrusion Detection Model based on Improved Support Vector Machine", Third International Symposium on Intelligent Information Technology and Security Informatics, 2010 IEEE.

[6] Z. Muda, W. Yassin, M.N. Sulaiman and N.I.Udzir,"Intrusion Detection based on K-Means Clustering and OneR Classification", 2011 IEEE.

[7] http://link.springer.com/chapter/10.1007\%2F978-3-64214400-4_50?LI=true\#

[8] http://www.personal.reading.ac.uk/ sis01xh/teaching/CY 2D2/Pattern3.pdf

[9] http://voyagememoirs.com/pharmine/2008/06/22/probabi listic-neural-network-pnn/

[10] S. Nascimento, B. Mirkin and F. MouraPires "A Fuzzy Clustering Model of Data and Fuzzy c-Means", Fuzzy Systems, the Ninth IEEE International Conference on May,2000.

[11] Zhou Mingqiang and Huang Hui, Wang Qian,"A Graphbased Clustering Algorithm for Anomaly Intrusion Detection", The 7th International Conference on Computer Science \& Education, July 2012. Melbourne, Australia.

[12] Sanjay Kumar Sharmai, PankajPande, Susheel Kumar Tiwari and Mahendra Singh Sisodia "An Improved Network Intrusion Detection Technique based on kMeans Clustering via NaIve Bayes Classificatio", IEEE-International Conference On Advances In Engineering, Science and Management March , 2012. 